\title{
El corazón de la mujer visto desde la Sociología con enfoque de equidad de género
}

\section{Women's hearts seen from a sociological point of view, with a gender equality focus}

\author{
Perla Olmos-Suárez ${ }^{\mathrm{a}, \mathrm{b}, \mathrm{c}, \mathrm{d}, \mathrm{e}}$
}

\author{
a Procuraduría General de la Nación, Contraloría General de la República, y del Ministerio de Agricultura y Desarrollo Rural en \\ equidad de género, desarrollo ambiental sostenible, política agraria y desarrollo rural, Bogotá, Colombia \\ b FAO, CORPOICA, Bogotá, Colombia \\ c Universidad Central, Bogotá, Colombia \\ ' Universidad Jorge Tadeo Lozano, Bogotá, Colombia \\ e Universidad IMPAU, Bogotá, Colombia
}

Recibido el 24 de julio de 2017; aceptado el 24 de noviembre de 2017

Disponible en Internet el 10 de enero de 2018

Por un episodio de mi vida, en el cual se involucró mi corazón desde la perspectiva biológica - orgánica, comencé a hacer una reflexión epistemológica de la más alta complejidad, pues desde la visión científica y operacional, el proceso está dado y se aplica con toda la rigurosidad de acuerdo con el adelanto de las ciencias y la tecnología para asegurar que la vida siga, con un corazón que vuelve a latir, como me dijera mi cardiólogo hemodinamista: "quedó su corazón como el de una quinceañera'. Este procedimiento fue realizado en la Fundación Cardioinfantil de Bogotá, donde tuve atención al órgano que late y le da vida a mi cuerpo siendo garante de una nueva salud que requiere de cuidados a aplicar con disciplina.

En momentos en que el corazón produce dolor, este se manifiesta físicamente de diversas formas, y también se tienen sensaciones de pérdida, temor, miedo y ausencia; sentimientos que involucran la siquis, el cerebro, y una parte poco estudiada e investigada del corazón en donde se perfile y se ausculte si es este el que siente y genera estas manifestaciones de sentimientos, o si por el contrario estos sentimientos se derivan o se desprenden sólo de la mente, sólo de ese monstruo o fenómeno como es el cerebro que ordena y equilibra el sentir o no sentir.

El corazón como un órgano fundamental y único para la vida, lo tenemos mujeres y hombres. Cuando falla se manifiesta con una dolencia o falencia similar para ambos, pues es una descompensación del cuerpo, del organismo del ser humano; y mujeres y hombres somos seres humanos, por lo tanto, el dolor y la sensación de pérdida se da con algunos grados de diferencia en cada uno por la misma diversidad

Correo electrónico: perlaolmos@hotmail.com 
y complejidad del organismo - lo biológico de unas y de otros.

En este artículo pretende hacerse un análisis desde el campo de la Sociología, frente a cómo y por qué se presentan los infartos en mujeres y hombres diferencialmente, siendo este un proceso orgánico y no social, pero con mucha incidencia en lo socio-cultural, con base en varios factores que inciden en la naturaleza del fenómeno, siendo distintos para los géneros, como las mujeres, los hombres y los LGBTI (sigla compuesta por las iniciales de las palabras lesbianas, gays, bisexuales y transexuales).

La dicotomía entre lo privado y lo público ha estado presente en el ayer, el ahora y el hoy, a través de la historia de la civilización occidental, prevaleciendo las mujeres en el espacio privado y en lo público el hombre. En este último espacio tiene valor todo el acontecer diario que explica y justifica la trayectoria o el devenir de las sociedades, de los grupos societales y el poder del hombre en su conjunto y accionar.

En lo privado está lo oculto, lo que no se da a conocer, lo íntimo, lo prohibido, lo secreto y es allí el lugar que la sociedad occidental le ha dado y justificado para las mujeres a través de un periodo de la historia de la humanidad, relegándose de ahí en perpetuo, el papel de las mujeres a la invisibilización, la exclusión y la negación de su derecho al desarrollo de su personalidad, fenómeno acompañado de la educación en todos sus niveles, que cumple una función determinante en la domesticación o alienación de las mentes y el conocimiento de mujeres y hombres con estereotipos que subvaloran el quehacer de las mujeres y su aporte al desarrollo de las sociedades.

Entonces el sitial de las mujeres, desde un período de la historia universal, la ocupante de ese espacio privado, lo magnifica la prensa, cuando se produce información por los distintos medios de comunicación escrita, televisión, radial entre otros. La otrora prensa del corazón o prensa femenina, como sinónimo de y para mujer, considerados y escritos sólo para ellas, ya que era sobre la vida privada y ese era el espacio sobre el cual las mujeres tenían acceso; por lo tanto, no era ni es en la actualidad de ningún valor científico, ni merece el rótulo o calificativo de textos serios, de formación, pero sí se construyó y construye el discurso alienante y excluyente para las mujeres.

Desde ese espacio privado las revistas, novelas escritas, radiales y televisivas llevan el mensaje claro para la perpetuación de una sociedad excluyente e inequitativa y desigual para o contra una población que tanto en el mundo como en Colombia representa más del $50 \%$ de sus habitantes (8.000.000 millones es la población mundial; de acuerdo con las Naciones Unidas y el Fondo mundial de Poblaciones, en Colombia se tienen más de 48 millones de habitantes) ${ }^{1}$.

Lo anterior permite entrar al fondo o al análisis de los factores de riesgo que producen en las mujeres la enfermedad cardiovascular (ECV) y el ataque cerebrovascular (ACV), de los cuales se hará una exposición somera desde la perspectiva sociológica y la equidad de género, y el porqué estas dos categorías que están unidas, permitirán entrar en este análisis del corazón, en la humanidad de las mujeres, sus causas, efectos y consecuencias.

Teniendo en cuenta que la primera causa de muerte en el mundo en ambos géneros son los ECV y ACV, tanto en los países desarrollados como en los tercermundistas, subdesarrollados o en vías de desarrollo, guardando las proporciones en cuanto a calidad de vida donde tiene un mayor peso la pobreza y la extrema pobreza que está presente en estos últimos países.

Según la Organización Mundial de la Salud - OMS, en el Informe del Estado Global de la Salud de abril de 2011, la mayor parte de las muertes en el mundo están causadas por enfermedades cardiovasculares, con un estimado de $29,82 \%$ que es el equivalente a 17 millones de personas de un total de 57 millones de muertes en el mundo ${ }^{2}$.

Igualmente, tener en consideración efectos de riesgo como los biológicos, la herencia y los factores psicosociales presentes en el momento de manifestación del hecho ha llevado a considerar las ECV como la mayor causa de mortalidad de las mujeres en el mundo con 8,6 millones de fallecimientos por año, cifra muy significativa y alarmante que requiere de propósitos científicos de alto nivel, pues está golpeando a una población que representa más del $50 \%$ de la población mundial.

Por su parte, la Organización Panamericana de la Salud (OPS) afirma que el $80 \%$ de las muertes por enfermedades cardiovasculares en el mundo, tienen lugar especialmente en los países cuya población es de ingresos bajos y medios, acotando que la incidencia es igual para hombres que para mujeres ${ }^{3}$.

La pobreza afecta más a las mujeres, tal y como lo demuestran investigaciones recientes en las que se evidencia que ésta constituye un factor de riesgo para el corazón femenino, con un $25 \%$ más de probabilidades de tener un ataque cardiaco frente a sus pares masculinos.

La población que vive en situación de pobreza y de extrema pobreza, marginalidad sin o con poco acceso a la educación y a los servicios básicos de sanidad-aseo, entre otros bienes necesarios pero no suficientes para llevar una vida digna, está expuesta en mayor medida a sufrir ECV, y entre esta población la más propensa a dichos episodios son las mujeres.

Aquí se juega el papel del poder como lo es la posición de menos privilegio en todos los aspectos en una sociedad patriarcal y masculinizada, agravando la situación cuando se trata de mujeres mayores en quienes las condiciones de calidad de vida disminuyen por su posición socio-económica, que no les permite acceder y tener una alimentación balanceada y mucho menos un ritual de ejercicios físicos, unido a muchos factores más como a recibir educación desde la niñez, tener una vivienda decente, y/o necesidades básicas satisfechas, esto último son condiciones que viven personas dentro de las familias pobres y en extrema pobreza, que en Colombia sustentan un porcentaje muy alto.

Este factor de pobreza incide en los altos riesgos de esta población comparada con personas y poblaciones que lo tienen todo; en este otro porcentaje de la población total al que se le facilita en parte la supervivencia con un rango de calidad de vida, la incidencia de afecciones cardiovasculares también tiene que ver con tres rangos: económico y social alto medio y bajo.

Con la evolución de las sociedades en la historia y los trascendentales periodos económico-sociales, se fueron perfilando las sociedades industrializadas después de la Primera y Segunda guerra mundial, lo cual trajo consigo la 
primera y segunda revolución industrial y el sistema económico del capitalismo, con sus diversas etapas y desarrollo progresivo del trabajo asalariado y el aprovechamiento de la plusvalía por los tenedores del gran capital, hasta llegar al neocapitalismo dentro de la sucesión de estadios de un sistema económico, basado en la propiedad privada, la empresa privada, la tierra privada y los demás factores de producción privados, la ganancia o plusvalía privada para los privados, dueños del capital financiero exportable pasando de la exportación de mercancías a la exportación de capitales en el mundo.

Se preguntarán, ¿qué tiene que ver esto con las enfermedades cardio- y cerebrovasculares? Tiene que ver, y mucho; a partir de la aparición en la palestra de un nuevo orden económico social y político como lo es el régimen capitalista, la producción de bienes de consumo y suntuarios necesitó mayor número de manos de obra, por un lado, y por otro niveles de pobreza, extrema pobreza e infinidad de población creciente, y explosión demográfica sin acceso y control a los factores de producción. Por algo hizo carrera la famosa frase "el tiempo es oro".

Estos periodos fueron propicios para que las mujeres entraran como fuerza de trabajo al mercado laboral, para contribuir con sus ingresos al hogar, devengando siempre un menor salario que el hombre, realizando la misma labor el mismo trabajo; según estimaciones del DANE (Departamento Administrativo Nacional de Estadística) y la CEPAL (Comisión Económica para América Latina y el Caribe) en Colombia las mujeres ganan entre un $30 \%$ y $25 \%$ menos que los hombres. Aquí no hay equidad de género, no hay valoración del trabajo de la mujer, el mercado está saturado de un ejército de reserva de mano de obra barata.

Aquí está el ejército de mujeres cuyo nivel de desempleo es mayor que el de los hombres, a pesar de su profesionalismo en igual o mayor medida que el hombre, con muchas empresas en las que se les exige producir más que un hombre; toda esta situación lleva intrínseco un proceso de cargas, responsabilidades y obligaciones. Allí aparecen la competencia, los retos, las metas, las presiones, las exigencias y el acoso laboral y otras veces sexual.

Todo ello es consecuencia de visibilizar la sociedad desde lo masculino, sin contemplar derechos fundamentales en igualdad de condiciones en salud a las mujeres; aquí se aprecia desde hace mucho tiempo la exclusión y no presencia del análisis de género en el campo de la salud. Cuando las mujeres trabajan por su doble y triple jornada, esta situación lleva a que sean propensas a tener episodios cardiovasculares relacionados con manejar altos niveles de estrés.

Entonces se puede considerar que los parámetros que suponían el infarto como una afección de hombres, hoy se ha invertido, cambio que puede asociarse a varios factores que han incidido en esta compleja situación para las mujeres; podríamos señalar y enumerar algunos, como por ejemplo, asumir una mayor responsabilidad, aumento de la jornada laboral o el trabajo en sí, desarrollo y logro de metas en el trabajo, obligaciones en el hogar y atención a la familia, niños, niñas, personas mayores, personas enfermas y hacia el compañeroo esposo.

Un factor de riesgo a considerar que reviste gran importancia es el estrés, que ya ha sido relacionado con las ECV. Este se manifiesta de diferentes maneras en las personas y está mucho más presente y afecta más a las mujeres que a los hombres. Lleva consigo las emociones fuertes ya sean positivas y/o negativas, muchas de las cuales están marcadas por situaciones o momentos angustiosos, dolorosos, que deprimen y golpean el corazón, la mente y la siquis, teniendo por tanto que reafirmar que afecta mucho más a las mujeres por factores y aspectos que se han venido tratando en este artículo, agregando otra realidad, y es que no es lo mismo la formación que la familia, el entorno, la educación en todos sus niveles y la sociedad en general le dan al hombre y a la mujer desde su gestación y todo el desarrollo personal cargados de estereotipos vigentes y actuantes para cada uno de los sexos. Aquí cabe anotar como se determina que el varón, el hombre es el fuerte, es el racional, es el analista, es el que no tiene sentimientos que pueda dejar aflorar o manifestar en público como el llorar, el sentir dolor por una pérdida, por una separación, por una muerte, por la falta de un trabajo, por la violencia, por la ausencia de amores, afectos, ternura, cariño, sueños, imaginarios por cumplir, todo ello le está condicionando a que no se manifieste. Es el hombre, es el súper hombre, el macho, el patriarca, el jefe, el que manda, el que detenta el poder, la autoridad, la disciplina y la resolución de los problemas de lo público, es ese hombre que se ha creado a través del tiempo; en cambio a la mujer le han sido asignado como si fuera connatural, todo lo que se le ha prohibido expresar al hombre, más el trabajo productivo fuera de casa.

Entonces, existe una dicotomía que se aprecia en la formación y que se ejemplifica en la sociedad, donde los roles, papeles o funciones de unas y otros van de acuerdo con lo que la sociedad define y quiere como comportamiento del uno y de la otra; este factor constituye la principal causa de las inequidades, las dominaciones, la exclusión, la subvaloración de la negación de los derechos de las mujeres frente a los derechos humanos, que son iguales ante la Ley respetando las diferencias de cada género. Esta situación se ha hecho cada vez más compleja para las mujeres.

Desde hace mucho tiempo podría afirmarse que desde siempre solo se puede rescatar un primer periodo de la historia de la humanidad, en el que las mujeres tuvieron un papel predominante en el periodo de la Comunidad primitiva consideradas sabias, sacerdotisas creadoras y transformadoras de cultura; su protagonismo se extendió y se hizo visible en el campo de la educación, la formación, la agricultura que como sedentarias por la misma división del trabajo y por poder parir se encargaban de las actividades del entorno y con ello dieron origen a este renglón tan importante para la alimentación, la seguridad y la soberanía alimentaria y se personaliza en la mitología con Isis, diosa de la agricultura, como su creadora.

También dieron vida a las manufacturas, responsables como diosas de la salud, dirigían las asambleas del pueblo, eran comunicadoras, socializadoras del conocimiento y los saberes, es decir estaban empoderadas como toda la comunidad donde hombres y mujeres eran diferentes pero iguales en sus derechos.

En la actualidad la falta de enfoque de género se materializa en la asignación de mayores tareas, compromisos, responsabilidades y presiones laborales, financieras y personales que hacen que se conviertan en súper-mujeres, produciendo estrés, ansiedad y angustia, haciendo que ellas 
mismas invisibilicen su cuerpo, mente y órganos como el corazón, incidiendo este descuido en desmedro de su propia salud y la vida misma.

Se puede inferir, sin temor a equivocarse, que toda esta situación de estrés que viven y acumulan las mujeres a través de cada día, se manifiesta como un factor más causante de las enfermedades cardiovasculares y los ataques cerebrovasculares. Sugieren los estudiosos del tema que el "estrés crónico" como un factor de riesgo importante de la enfermedad cardiovascular, se evalué de forma rutinaria y se gestione en forma efectiva como los otros factores de riesgo importantes, causantes de la enfermedad coronaria como el fumar, la hipertensión y la diabetes que se posesione en ese mismo renglón y con la misma importancia el estrés (Ahmet Tawakol, codirector del programa de imágenes cardiacas del Hospital General de Massachusetts en Boston).

Investigaciones y estudios llevados a cabo por universidades y profesionales en la materia, afirman que hay un espacio en el cerebro llamado "pelota de estrés", que es una amígdala constituida por una "colección de células nerviosas en el cerebro en forma de pelota de fútbol americano, que influye en la forma en que respondemos al estrés".

Reduciendo los niveles de estrés, los episodios cardiovasculares y cerebrovasculares tendrían que descender específicamente en las mujeres.

El 90\% de las mujeres tienen uno o más factores de riesgo para desarrollar enfermedad cardiaca. Los otros factores de riesgo son los que han venido siendo considerados para los hombres por tratarse de una enfermedad para hombres. Como ya se ha anotado en párrafos anteriores, esos factores tienen que ver con lo biológico, lo orgánico, y a estos se les suma una nueva etapa en la mujer, la menopausia, que por la ausencia de la generación de estrógenos constituye otro factor de riesgo, unido en un todo con lo sicosocial.

Otra arista que acompaña esta situación es el desconocimiento y la falta de entrenamiento de profesionales de la salud en general, para escuchar a las mujeres y considerar sus síntomas y manejo cuando van a consulta o son llevadas de urgencia, para que estos no sean confundidos con otras patologías o enfermedades de otros órganos, siendo este mi caso, cuando realizaron un diagnóstico erróneo en urgencias que puso en juego mi vida: electrocardiogramas normales, diagnóstico inicial manejado con relajante muscular, egreso hospitalario que requirió reingreso a urgencias en otra institución a las pocas horas; nuevos electrocardiogramas no conclusivos, sin evidencia de un evento cardiaco, un ecocardiograma que orientó el diagnóstico cardiovascular y aplicación de medicamentos con efectos secundarios que nuevamente pusieron en riesgo mi vida. Mi gran deseo de vivir y un giro en el desenlace, con esa frase al final del episodio crítico y que transcribí desde el primer párrafo de este artículo: "quedó su corazón como el de una quinceañera", con base en esa experiencia personal, fue lo que trazó parte del camino para escribir este artículo. Quiero también hacer con él, un llamado de atención a los profesionales de la salud especialmente a los del corazón, sobre la dimensión que cubre un error en el diagnóstico y sobre el tratamiento desde una perspectiva o enfoque de género, por lo ya anotado sobre los síntomas diferenciales de acuerdo con el género y el sexo en que se da el evento cardiovascular o el ataque cerebrovascular.
En las consultas bibliográficas se afirma que los equipos de salud en el mundo no están preparados para abordar, asumir y tratar de forma segura y con la rapidez que se necesita diagnosticar eventos cardiovasculares en mujeres, que se presenta en la misma proporción o cantidad que la de los hombres. Por ello es necesario tomar en serio los síntomas de ECV en las mujeres, pues existe un número de éstas con incidencia cardiaca a sus pares, que de existir errores en el diagnóstico, contribuyen a un peor pronóstico que conduce a desenlaces fatales por la falta grave de no aplicar un enfoque de género que permita visibilizar que la persona que se atiende es una mujer, quien no se puede medir ni diagnosticar como si presentara las manifestaciones, condiciones y síntomas cardiacos de un hombre. ¿Cuántas mujeres han sufrido un infarto después de un error en el diagnóstico por ignorar los factores de riesgo para su corazón?

El hecho real y contundente es que más hombres consultan al cardiólogo, a otro especialista o a un médico general, debido a su preocupación cardiaca, cuando sienten que les duele el pecho u otra manifestación que pueden percibir que puede comprometer su corazón; no ocurre así en igual proporción con las mujeres, en cuanto a visitas a un especialista especialmente cardiólogo, internista y médico general porque las situaciones para ella no son alarmantes ni identifica que esto sea previo a una ECV, como tal, y el médico o especialista tampoco lo hacen, por tanto esta situación en el mundo y en Colombia, requiere de una mirada, una visión, una percepción, un conocimiento, un pronóstico confiable, y llama la atención a habilitar en este campo una lectura de género, que tenga en cuenta el papel, el rol, la posición y condición de las mujeres en la sociedad, que lleva a considerar su vida y su agenda de la triple jornada que realiza, sus responsabilidades, sus compromisos en el hogar, en el entorno, en el trabajo productivo y en la sociedad o círculo social donde acontece su devenir diario.

Si se tiene en cuenta que las enfermedades cardiovasculares son la causa principal de muerte de las mujeres en el mundo, es más que suficiente razón para que se adelanten investigaciones profundas en las que la equidad de género sea el centro y esta categoría de análisis social se convierta en eje transversal, que dé cuenta de la verdadera incidencia numérica en las mujeres afectadas y así se adelanten procesos de atención, prevención, cuidado y seguimiento que conlleven diagnósticos reales y ajustados a la realidad biológica y social de esta población.

Las investigaciones que se desarrollen sobre este tópico o situación de las mujeres frente a las ECV, deben partir de la visibilización de estas como población vulnerable de ataques cardiacos, descubriendo y atendiendo su sintomatología atípica en consideración a las de sus pares, de allí que dichas investigaciones se deben centrar en el cómo, cuándo, dónde, por qué, tiempo, espacio, lugar geográfico, medio social, cultural, económico, ambiental, calidad y posición, mínimo vital, frecuencia en la muestra de una prueba que se pueda generalizar o por lo menos aplicar en donde se encuentren esas mismas condiciones y categorías investigadas.

¿Por qué a pesar de constituirse como la primera causa de muerte por enfermedad cardiovascular en mujeres en el mundo, se sigue considerando que la enfermedad cardiovascular es una enfermedad con "rótulo de hombre"? 
En la actualidad los científicos han tratado de diseñar una propuesta para desarrollar, recomendar y aplicar a cada caso una mirada diferente en la búsqueda de una mayor sensibilización y preocupación por visibilizar este fenómeno o situación de la ECV en las mujeres, adelantando campañas de divulgación tanto para el gremio de profesionales en este campo, como para la sociedad en su conjunto y específicamente hacia las mujeres, quienes en su mayoría desconocen la sintomatología que se da en ellas como alerta prodrómica de una ECV, entidad que generalmente se confunde y subvalora como manifestación de otras patologías, por ese error de auscultar y mirar a las mujeres con síntomas masculinizados de un infarto, en búsqueda de manifestaciones y síntomas de estos fenómenos cardiacos de igual manera que en los hombres cuando la realidad de los síntomas de una enfermedad cardiovascular en las mujeres es completamente diferente. De allí la importancia del manejo de la equidad de género como categoría de análisis social, para comprender el porqué de estas manifestaciones diferenciadas entre mujeres y hombres frente a un problema del corazón.

Para concluir, se insiste en que es urgente y necesario tener presente y aplicar el análisis con un enfoque de género que transversalice estudios, investigaciones y la práctica médica, desde el nivel general hasta los especialistas y específicamente los encargados del corazón de mujeres y hombres de manera intergeneracional, lo cual representa una ganancia para las mujeres, la ciencia, la sociedad y las estadísticas que se nutrirán de una información desagregada por sexos y por factores de riesgo, con datos importantes sobre cómo se enferman los hombres y las mujeres y cuáles son los síntomas diferenciales de estas enfermedades y de las muertes por ECV y ACV. Esto no ha venido haciéndose ni atendiéndose rigurosamente en su verdadera dimensión, constituyéndose en un sesgo o brecha de género que ha incidido negativamente en los diagnósticos y pronósticos de una enfermedad catalogada silenciosa en las mujeres, pues han sido invisibilizadas o ignoradas por falta de conocimiento sobre su salud.

Registro con complacencia que en algunos países de Europa, especialmente en España, se esté visibilizando el paradigma de equidad de género, estando presente en conferencias, reuniones, talleres, trabajos y encuentros en los que se dan cita profesionales de la salud en el mundo preocupados por el acontecer de las ECV y ACV en las mujeres. Así, se le da suma relevancia a este tema con un enfoque interdisciplinario, para lo cual se ha tenido en cuenta la propuesta hecha por la Organización Mundial de la Salud, que considera el conjunto, la variedad y la complejidad que se tienen en cuenta por las diferencias de género en distintos contextos y reafirman la importancia del enfoque de género como eje transversal en el campo de la salud.

\section{Conflicto de intereses}

Ninguno.

\section{Bibliografía}

1. Demografía y población DANE. Disponible en: https://www.dane. gov.co/index.php/estadisticas-por-tema/demografia-ypoblacion

2. Informe sobre la situación mundial de las enfermedades Disponible en: www.who.int/nmh/publications/ncd_report summary_es.pdf

3. La salud en las Américas Organización Panamericana de la Salud, Pan American Health Organization. Disponible en: https: / / books.google.com.co / books?isbn=9275315876 